\title{
An Apparatus for Measuring the Piezoresistivity of Semiconductors
}

\author{
R. F. Potter ${ }^{2}$ and W. J. Mc Kean
}

\begin{abstract}
A detailed description is given of an apparatus and procedure designed to measure the piezoresistive effect in semiconductors over an extended temperature range. A tensile force up to 1 kilogram can be applied to the sample by means of a calibrated beam balance. The apparatus has been used for measurements on indium antimonide over the range $78^{\circ} \mathrm{K}$ to $300^{\circ} \mathrm{K}$, and tensile stresses of the order of $5 \times 10^{7}$ dynes per square centimeter can be applied to samples that are cut in a special manner.
\end{abstract}

In modern solid-state physics, phenomena such as electrical conduction, Hall effect, and optical absorption have been studied extensively because of their direct connection with a well-developed theory of semiconductors. More recently several other effects have been receiving an increasing amount of attention by both the theorist and experimentalist; some of these are cyclotron resonance, photoelectromagnetic effect, magnetoresistivity, and piezoresistivity.

The latter parameter has been studied for some time in connection with metals, but it is only in comparatively recent years that large anisotropic changes in the resistivity with applied stress were measured for single crystals with cubic symmetry. Smith [1] ${ }^{3}$ measured large effects for both $n$ - and $p$-type crystals of germanium and silicon at room and liquid-nitrogen temperatures, thus adding further to the evidence that the energy surfaces for electrons in these crystals are not spheres but ellipsoids. More recently, Morin, Geballe, and Herring [2] reported on measurements in which the piezoresistive effect was produced with compressive stress. Herring [3] has discussed the above phenomena in terms of a theory of "simple manyvalley" semiconductors, which seems to describe the conduction bands for silicon and germanium, and possibly several III-V compound semiconductors.

The piezoresistivity coefficients are the elements of a fourth-rank tensor; the form has been given by Smith [1] for cubic crystals of the $O_{h}$ and $T_{d}$ classes, which apply to crystals of the diamond and zinc blende lattices, respectively, and is given by

$$
\Pi=\left|\begin{array}{cccccc}
\Pi_{11} & \Pi_{12} & \Pi_{12} & 0 & 0 & 0 \\
\Pi_{12} & \Pi_{11} & \Pi_{12} & 0 & 0 & 0 \\
\Pi_{12} & \Pi_{12} & \Pi_{11} & 0 & 0 & 0 \\
0 & 0 & 0 & \Pi_{44} & 0 & 0 \\
0 & 0 & 0 & 0 & \Pi_{44} & 0 \\
0 & 0 & 0 & 0 & 0 & \Pi_{44}
\end{array}\right|,
$$

1 This research was supported by the United States A ir Force through the Air Force Office of Scientific Research of the Air Research and Development Command under Contract CSO $670-53-12$.

2 Present Address-U.S. Naval Ordnance Laboratory, Corona, Calif.

3 Figures in brackets indicate the literature references at the end of this paper. a form quite analogous to that for the elastic constants. The II-constants are defined as follows:

$$
\begin{array}{ll}
\Pi_{11}=\Pi_{11,11} \\
\frac{\Delta \rho_{\alpha \beta}}{\rho_{o} X_{i j}}=\Pi_{\alpha \beta i j} & \Pi_{12}=\Pi_{11,22} \\
\Pi_{44}=\Pi_{12,12 .}
\end{array}
$$

$X_{i j}$ is the applied stress, and $\rho$ is the resistivity, where the cubic axes are taken as the reference axes.

The change of resistance in a material is measured when a stress is applied in a given direction to a crystal while a constant current flows at a given angle to the stress direction. As in the case of elastic constants for a cubic material, three independent measurements must be made to determine the elements of the tensor of formula 1. Smith measured the longitudinal and the transverse effects in two samples of different orientations. The fourth determination served as a check on the other three. The longitudinal effect is defined as the relative change observed in the resistance per applied stress when both current and stress have the same direction. The transverse effect is measured when current and stress are perpendicular to each other.

In converting the resistance to resistivity it is necessary to consider the contraction due to strain. The piezoresistivity coefficients are given by the formulas

$$
\begin{array}{ll}
\Pi_{11}^{\prime} & =\frac{\Delta \rho_{11}^{\prime}}{\rho_{0} X_{11}^{\prime}}=\frac{\Delta R_{11}^{\prime}}{R_{0} X_{11}^{\prime}}-\left(S_{11}^{\prime}-S_{12}^{\prime}-S_{13}^{\prime}\right) \quad \text { (long.) } \\
\Pi_{12}^{\prime}=\frac{\Delta \rho_{22}^{\prime}}{\rho_{0} X_{11}^{\prime}}=\frac{\Delta R_{22}^{\prime}}{R_{0} X_{11}^{\prime}}+\left(S_{11}^{\prime}-S_{12}^{\prime}+S_{13}^{\prime}\right) \quad \text { (trans.), }
\end{array}
$$

where $S_{i j}^{\prime}$ are the elastic compliances for the orientation used. Values for $S_{i j}$ have been determined for silicon [4], germanium [4], and indium antimonide [5]. The $\Pi_{i j}^{\prime}$ are related to the elements of the tensor (1) by

$$
\begin{aligned}
& \Pi_{11}^{\prime}=\Pi_{11}-2 \Gamma \Pi^{\prime} \\
& \Pi_{12}^{\prime}=\Pi_{12}+\gamma \Pi^{\prime},
\end{aligned}
$$


where $\Pi^{\prime}=\Pi_{11}-\Pi_{12}-\Pi_{44}$, and $\Gamma$ and $\gamma$ are orientation factors related to the direction cosines of axes along the length and width of the sample as follows:

$$
\begin{gathered}
\Gamma=l_{1}^{2} m_{1}^{2}+n_{1}^{2} m_{1}^{2}+n_{1}^{2} l_{1}^{2}, \\
\gamma=l_{1}^{2} l_{2}^{2}+m_{1}^{2} m_{2}^{2}+n_{1}^{2} n_{2}^{2} .
\end{gathered}
$$

A program was recently undertaken at the Bureau to study the piezoresistivity of the cubic semiconductor indium antimonide. (Concurrent studies were made of the magnetoresistivity of this material.)

Because of the nature and properties of the material, several considerations had to be taken in the design of an apparatus to measure piezoresistance. First was the matter of temperature. Reasonably pure indium antimonide is intrinsic at room temperature, whereas it is certainly extrinsic at $78^{\circ} \mathrm{K}$; therefore, the temperature range down to that of liquid nitrogen had to be the minimum range studied. Indium antimonide is extremely brittle; a suitable method of clamping the sample had to be devised in order that known stresses could be applied along the axes of the sample. It is felt that the resultant design more than adequately solved the special problems posed by these properties.

A tensile-stress system was selected as being the simplest to apply and to measure in a closed system. As shown in figure 1, a balance beam. (S) graduated in 100-g intervals up to $1 \mathrm{~kg}$ was devised and along which a weight (U) could be moved. Counterbalance weights (J and $\mathrm{K}$ ) could be adjusted so that the beam balanced with zeroload. The load was transmitted to the sample by means of a thin piano wire $(\mathrm{N})$, which was coaxial with an inner stainless-steel tube (Q), which in turn was coaxial with the outer stainlesssteel tube (P). As the load was applied or removed, the wire suffered a plastic strain, which could be compensated by moving the fulcrum $(\mathrm{R})$ up or down by means of a nut (D). A given load could be applied or removed quickly by turning the handle (C), which activated the attached cam arrangement.

Figure 2 shows a schematic cross section of the sample holder. The problem of applying pure tensile stress to the sample was solved as follows. A magnetostrictive machine was used for cutting flat specimens into a suitable form for resistivity measurements by the standard four-probe method; a special die was made to produce samples of the shape shown in figure 3. Each end of the sample was placed in a clevis with a pin through the eye (see fig. 2). The upper clevis $(\mathrm{L})$ was attached to the piano wire; and the bottom clevis (S) was the upper part of a screw. A spherical-shaped nut (V) on this screw seated in a mating socket allowed the sample to aline its axis in the direction of the applied tensile stress. This arrangement also permitted further adjustment in the vertical position of the sample. It was eventually

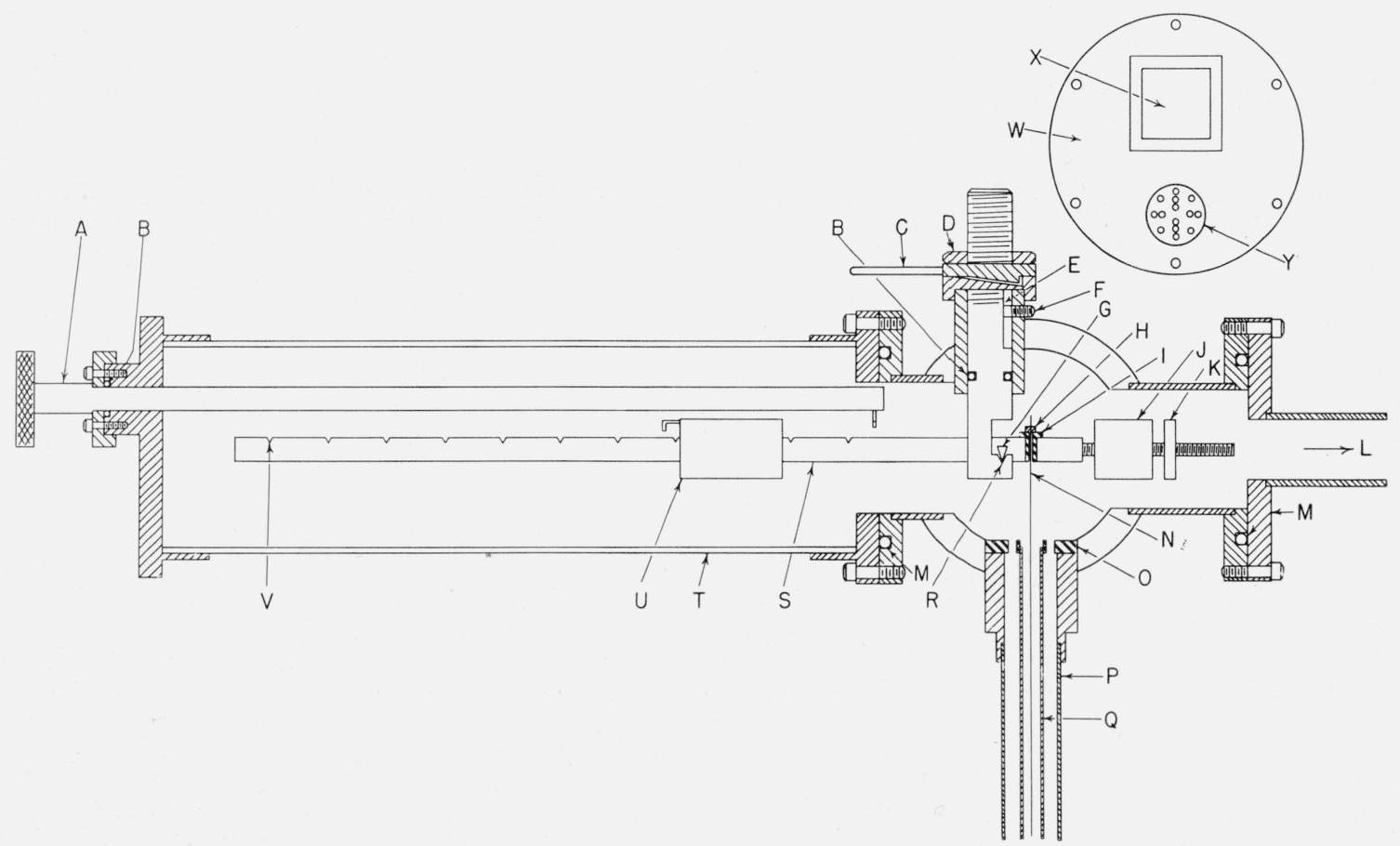

FIgURE 1. Schematic drawing of the balance-beam mechanism.

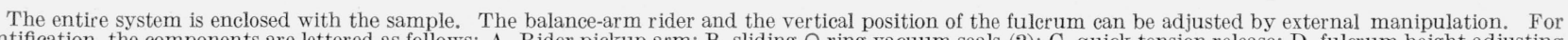

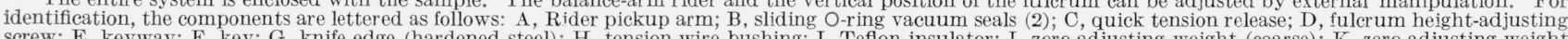

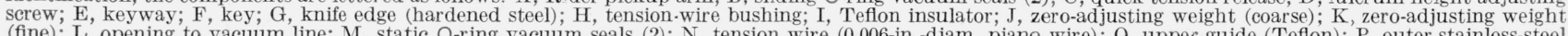

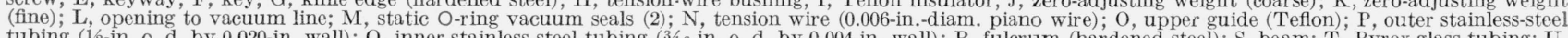
tubing (1/2-in. o. d. by 0.020 -in. wall); Q, inner stainless-steel tubing (3,16-in, o. d. by 0.004 -in. wall); R, fulcrum (hardened
rider; V, detents (11, 100 g apart); W, front cover for balance mechanism; X, glass inspection port; Y, 14-lead hermetic seal. 


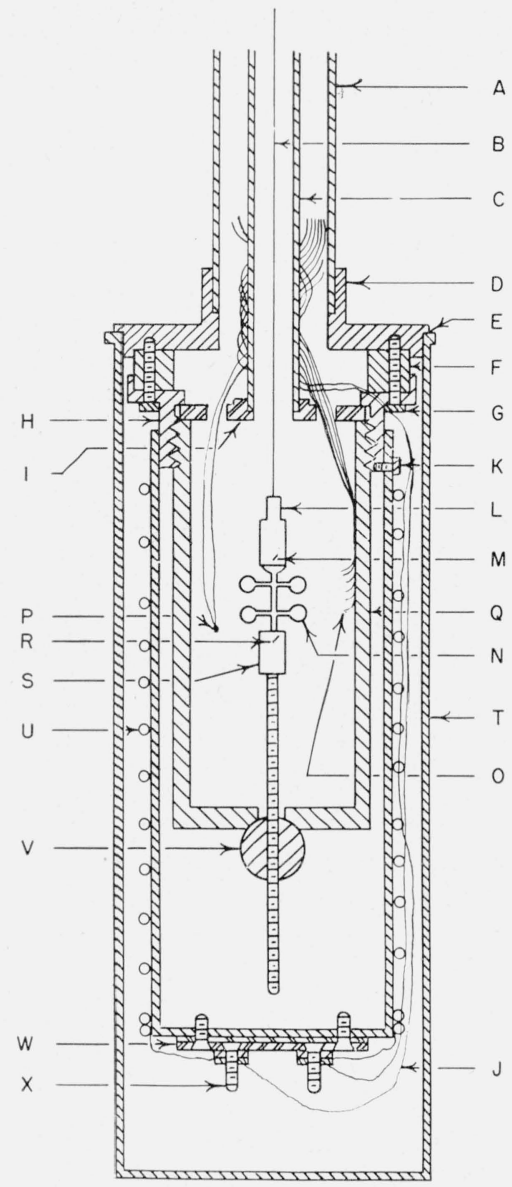

FIGURE 2. Schematic drawing of the sample and holder.

By means of the pins through the sample ends, the sample axis lines up with the applied stress. For identification the components are lettered as follows: A Outer stainless-steel tubing (1/2-in. 0 . d. by 0.020 -in. wall); B, tension wire $(0.006-$ in.-diam. piano wire); C, inner stainless-steel tubing ( $3 / 16$-in. o d by 0.004 -in. wall); D, top; E, vacuum-tight removable solder joint (Wood's metal); F, lava thermal insulator; G, stainless-steel screws; H, frame holder; I, lower guide (Teflon); J, heater leads; $K$, heater retaining screw: $L$, upper clevis; $M$, clevis pin; N, sample; $O$, contact leads; $P$, thermocouple; $Q$, frame; $R$, clevis pin; $\mathrm{S}$, lower clevis; T, outer can; U, heater;'V, lower spherical ad justing nut; W, Teflon insulation; $\mathrm{X}$, heater binding posts.

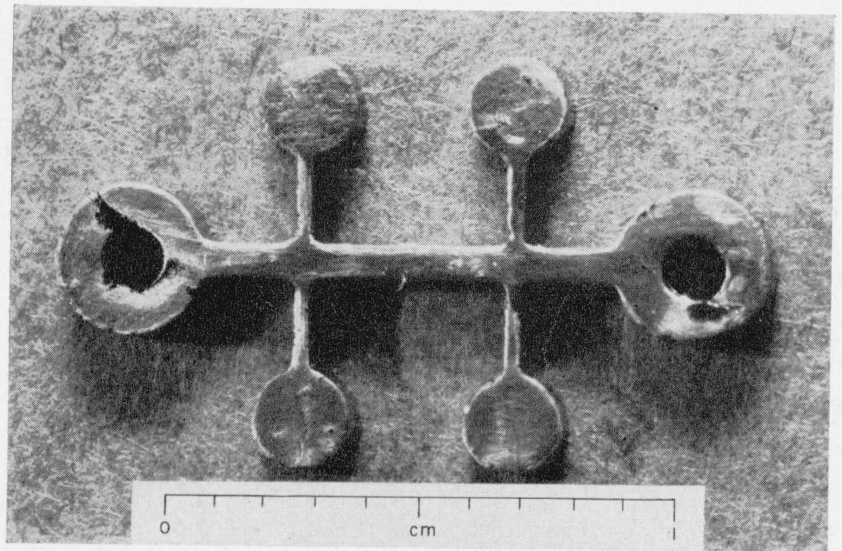

FIGURE 3. Typical sample used with the apparatus. determined that a sample of this type and size (approximately $10^{-2} \mathrm{~cm}^{2}$ cross section) can be safely recycled many times with loads up to $500 \mathrm{~g}$.

An aluminum shield may be placed around the sample holder. On the outside of this shield is wound a Kanthal wire heater (U) for temperature control. A brass can (T) was soldered in place with Wood's metal; the entire interior could be evacuated or filled with a gas of suitable pressure. For use at temperatures between $78^{\circ}$ and $300^{\circ} \mathrm{K}$ a Pyrex envelope was placed about the sample can and the stainless-steel tube (see fig. 4). This envelope was attached to a roughing pump, and the pressure of the gas inside varied from $1 \mathrm{~atm}$ to a few microns of mercury; this allowed the heat leak from the sample to be controlled when a suitable refrigerant bath was placed around the envelope. (For lower temperatures, e. g., $4^{\circ}$ or $20^{\circ} \mathrm{K}$, the envelope can be replaced by a Dewar designed for holding liquid helium or liquid hydrogen. Figure 4 shows the assembled apparatus with the Pyrex envelope.

Measurements on both $n$ - and $p$-type indium. antimonide have been made at temperatures between $78^{\circ}$ and $300^{\circ} \mathrm{K}$, and the results are reported elsewhere [6]. The resistances were measured by the potentiometric method, using a type $\mathrm{K}-2$ potenti-

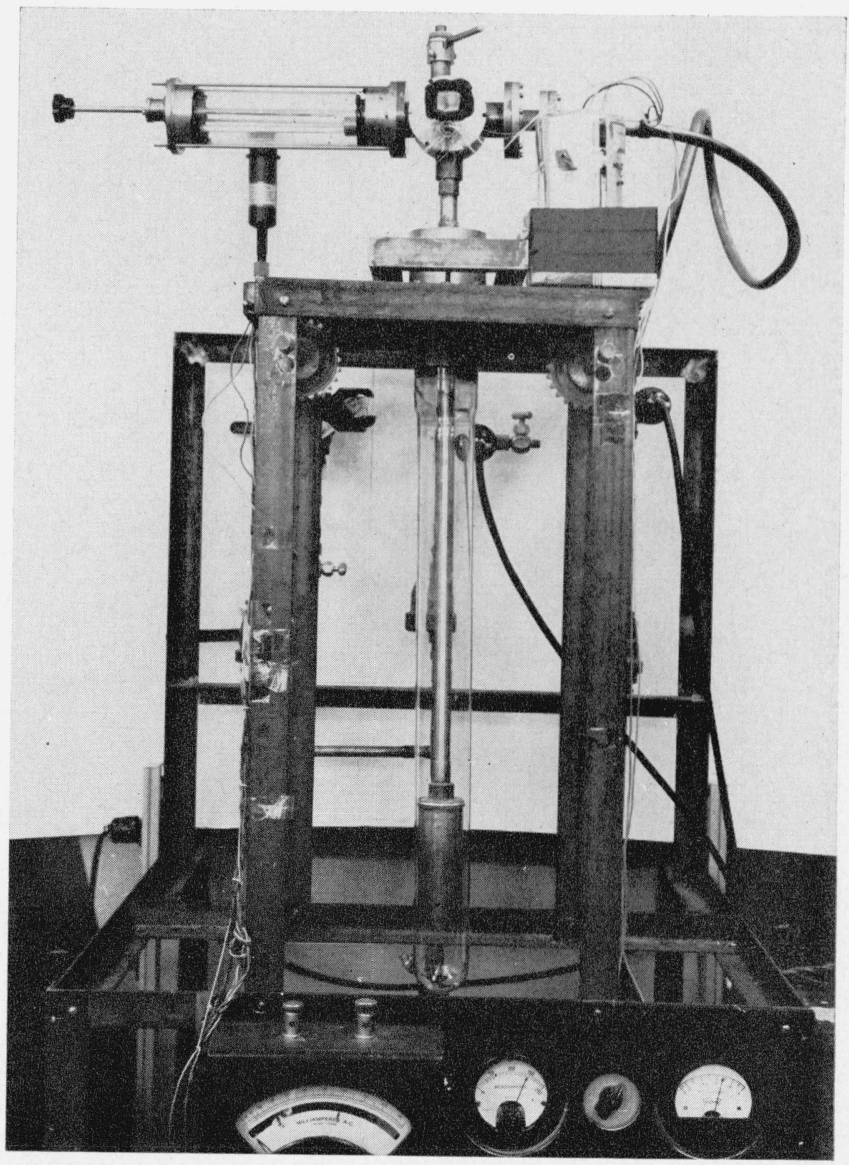

Figure 4. Assembled apparatus with the Pyrex envelope.

The frame supports associated vacuum lines, valves, meters, and pressure gages. It can also support a double Dewar for measurements in the liquid-helium range. 
ometer a breaker-type d-c amplifier in place of the galvanometer. The output of the amplifier was fed to a driven chart recorder, so that as the load was changed the incremental change in resistance was recorded by the unbalanced potentiometer. Checks were made to insure that currents due to the unbalance has negligible effects. Effects due to changes in the ambient temperature were observed on the recorder and the necessary corrections made.

Samples were prepared in the following manner. Disks $2 \mathrm{~mm}$ thick and about 1 in. in diameter were sawed from single-crystal material with (001) axes normal to their flat surfaces. These were lapped to a thickness of 1.0 to $1.1 \mathrm{~mm}$, taking care that both sides were parallel. The disks were then mounted on plate glass with glycol phthalate. An X-ray Laue back-reflection photograph was taken normal to the exposed side of each disk and analyzed. From the analysis the axes of the specimens were selected to lie along a particular orientation. A rotary milling table was used on the magnetostrictive machine so that the sample could be accurately oriented with respect to the tool. Indium electrodes were soldered to the four side arms and along the sides of the dumbbell-shaped ends. For the longitudinal effect, the end electrodes were used for the current leads, and two side arms were used for the potential leads. For the transverse measurements, the areas between a pair of arms on each side of the specimen were copper plated by applying a copper-sulfate solution momentarily and then rinsing, taking care that no short circuit across the sample occurred. The measurements were made by having one current lead and one potential lead opposite to another such pair while the stress was applied along the sample axis. As pointed out by Smith [1], the current lines are not straight across the sample. Hence, a correction must be made if the piezoresistive coefficients deduced from transverse and longitudinal measurements show appreciable differences.

It is felt that the apparatus has solved the special problems that are inherent in this type of measurement and a material like indium antimonide. Determination of the longitudinal effect by means of this apparatus if used in conjunction with hydrostatic-pressure measurements (cf. Long [7], Keyes [8], or Bridgman [9]) would eliminate the need for the transverse measurements. With a slight modification of the sample holder to insure a fixed orientation of the sample with respect to a magnetic field, one could also determine changes of the Hall coefficient and magnetoresistivity caused by applied stresses.

[1] C. S. Smith, Phys. Rev. 94, 42 (1954).

[2] F. S. Morin, T. H. Geballe, and C. Herring, Phys. Rev 105, 525 (1957).

[3] C. Herring, Bell System Tech. J. 34, 237 (1955).

[4] H. J. MeSkimin, J. Appl. Phys. 24, 988 (1953).

[5] R. F. Potter, Phys. Rev, 103, 47 (1956)

[6] R. F. Potter, Bul. Am. Phys. Soc. [II] 2, 121 (1957); Phys. Rev. 108 (1957)

[7] D. Long, Phys. Rev. 99, 388 (1955).

[8] R. W. Keyes, Phys. Rev. 99, 490 (1955).

[9] P. W. Bridgman, Proc. Am. Acad. Arts Sci. 60, 305(1925)

Washington, May 6, 1957. 